Теорія Ймовір. та Матем. Статист. Вип. 75, 2006
Theor. Probability and Math. Statist.

No. 75, 2007, Pages 189-199 S 0094-9000(08)00725-4

Article electronically published on January 25, 2008

\title{
ASYMPTOTIC QUANTIZATION ERRORS FOR UNBOUNDED QUANTIZERS
}

UDC 519.21

\author{
M. SHYKULA
}

\begin{abstract}
We consider non-uniform scalar quantization for a wide class of unbounded random variables (or values of a random process sampled in time). Asymptotic stochastic structures for quantization errors are derived for two types of quantizers when the number of quantization levels tends to infinity. The corresponding results for bounded random variables are generalized. Some numerical examples illustrate the rate of convergence.
\end{abstract}

\section{INTRODUCTION}

Quantization of continuous random signals (or random variables and processes) is an important part of digital representation of analog signals for various coding techniques (e.g., source coding, data compression, archiving, restoration). Quantization aims at representing a realization of a continuous in value and/or time random process (or signal) by a finite-dimensional vector. The most comprehensive overview of quantization problems can be found in [6] (for later references, see [5]). A quantizer introduces some distortion into the data and the problem of evaluation of this distortion arises. A number of authors estimate distortion measures for quantizers (see, e.g., [14] and [8]). A class of asymptotically optimal quantizers with respect to an $r$ th-mean error distortion measure is considered in [4] (see also 3, 13]). A different approach for uniform scalar quantization is developed in [12, where the correlation properties of a Gaussian process are exploited to evaluate the asymptotic behavior of the random quantization rate for uniform quantizers. General quantization problems for Gaussian processes in infinite-dimensional functional spaces are considered in [10.

Mapping of a realization of a continuous in value and/or time process into a finitedimensional vector requires both discretization in time (or sampling) and amplitude (or quantization). The problems of optimal sampling for spline approximation are studied in [11. We consider quantization of a random variable, which is the value of a random process at a fixed sampling point. In this paper we derive the stochastic structure of the asymptotic quantization error for a general class of quantizers when the number of quantization levels tends to infinity. Let $X$ be a continuous random variable with a density function $f(x), x \in \mathbb{R}$. Following Bennett's notations (see [1]), the $N$-level companding quantizer (also called compander) $Q_{N, G}(x)$ is defined as

$$
Q_{N, G}(x)=G^{-1}\left(Q_{N, U}(G(x))\right),
$$

2000 Mathematics Subject Classification. Primary 60G99; Secondary 94A29, 94A34.

Key words and phrases. Non-uniform scalar quantization, random process, stochastic structure. 
where $G: \mathbb{R} \rightarrow(0,1)$ is onto and increasing, and $Q_{N, U}$ is the $N$-level uniform quantizer on $(0,1)$,

$$
Q_{N, U}(y)= \begin{cases}\frac{n}{N}-\frac{1}{2 N} & \text { if } y \in\left(\frac{n-1}{N}, \frac{n}{N}\right], n=1, \ldots, N-1, \\ 1-\frac{1}{2 N} & \text { if } y \in\left(\frac{N-1}{N}, 1\right) .\end{cases}
$$

Note that the inverse function $S:=G^{-1}$ of $G$ provides a transformation from a uniform quantization to a non-uniform one. The quantization intervals of the companding quantizer $Q_{N, G}$,

$$
I_{1, N}=\left(-\infty, S\left(\frac{1}{N}\right)\right], \quad I_{n, N}=\left(S\left(\frac{n-1}{N}\right), S\left(\frac{n}{N}\right)\right], \quad n=2, \ldots, N-1,
$$

and

$$
I_{N, N}=\left(S\left(\frac{N-1}{N}\right), \infty\right),
$$

and the corresponding quantization levels,

$$
u_{n, N}=S\left(\frac{n}{N}-\frac{1}{2 N}\right), \quad n=1, \ldots, N
$$

set up a design $D_{N}=D_{N, G}, N \geq 1$. Let $g(x)$ define the probability density function corresponding to $G(x)$, i.e., $G(x)=\int_{-\infty}^{x} g(v) d v, x \in \mathbb{R}$. We call $g(x)$ the quantization density function. In what follows we consider a sequence of regular designs

$$
D_{N}(g)=D_{N, G}(g), \quad N \geq 1,
$$

generated by a positive continuous $g(x), x \in \mathbb{R}$,

$$
\int_{-\infty}^{S(n / N)} g(x) d x=\frac{n}{N}, \quad n=1, \ldots, N-1,
$$

and

$$
\int_{-\infty}^{S(n / N-1 /(2 N))} g(x) d x=\frac{n}{N}-\frac{1}{2 N}, \quad n=1, \ldots, N .
$$

Note that $g(x)$ characterizes the distribution of quantization levels. It follows from (11) that $G(x)$ provides a transformation from a non-uniform quantization of the random variable $X$ to a uniform quantization of a random variable $G(X)$ with values in $(0,1)$, i.e., $G\left(Q_{N, G}(x)\right)=Q_{N, U}(G(x))$. Henceforth, let $\stackrel{d}{\rightarrow}$ denote convergence in distribution.

Some problems on asymptotically optimal companding quantization are discussed in [1. For example, for the $r$ th-mean error distortion measure

$$
D\left(Q_{N, G}(X)\right)=\mathrm{E}\left|X-Q_{N, G}(X)\right|^{r}, \quad r>0,
$$

it is shown in [1] that

$$
\lim _{N \rightarrow \infty} N^{r} D\left(Q_{N, G}(X)\right)=\frac{1}{(r+1) 2^{r}} \int_{\mathbb{R}} \frac{f(x)}{g(x)^{r}} d x=C_{r}>0 .
$$

It follows by Hölder's inequality that the right-hand side of (3) is minimal if and only if $g(x)=f(x)^{1 /(r+1)} / \int_{\mathbb{R}} f(z)^{1 /(r+1)} d z$ (cf. [3]). For a random variable $X$ with values in a finite interval, the stochastic structure of the asymptotic normalized quantization error and asymptotic properties of the corresponding additive noise non-uniform quantization model are derived in [13. Probability densities of the asymptotic vector quantization errors are studied in [7. In our paper we obtain the stochastic structure of the asymptotic non-uniform scalar quantization errors for a random variable when the support of the density is not necessarily finite (unbounded quantizer). 
The paper is organized as follows. In Section 2 we consider two types of non-uniform scalar quantizers. For these quantizers we find the stochastic structures of asymptotic normalized errors for non-uniform quantization of random variables. Section 3 provides some examples and numerical experiments to illustrate the rate of convergence in the obtained results. In Section 4 proofs of the statements in the previous sections are given.

\section{RESUlTS}

In this section we consider the stochastic structure of the asymptotic quantization error for two non-uniform companding quantizers. For a random variable with values in a finite interval the stochastic structure of non-uniform quantization error is derived in [13] asymptotically when the number of quantization levels tends to infinity. Henceforth, let $X$ be a continuous random variable with density function $f(x), x \in \mathbb{R}$, not necessarily with bounded support. Let $g(x)$ be a quantization density function. From now on we assume that the density functions $f(x)$ and $g(x)$ have the same support. Various conditions on the density functions of quantized random variables and quantization density functions are considered in a number of quantization papers, mostly in the applied literature (see, e.g., 7]). We introduce the following conditions on $f(x)$ and $g(x)$ :

$\left(A_{1}\right)$ The quantization density function $g(x), x \in \mathbb{R}$, is positive and uniformly continuous.

$\left(A_{2}\right)$ There exists $C>0$ such that $f(x) \leq C g(x)$ for every $x \in \mathbb{R}$.

$\left(A_{3}\right)$ The density function $f(x), x \in \mathbb{R}$, is continuous.

For example, the assumptions $\left(A_{1}\right)-\left(A_{3}\right)$ hold if the density functions $f(x)$ and $g(x)$ are Gaussian with identical parameters.

Let $G(x)$ be a distribution function corresponding to $g(x)$. For $X$ and $G(x)$ recall that $Q_{N, G}(X)$ denotes the $N$-level companding quantizer with quantization design

$$
D_{N}=D_{N}(g), \quad N \geq 1
$$

(cf. (10). In the following theorem, the stochastic structure of the normalized nonuniform quantization error $Z_{N, G}(X)=N\left(X-Q_{N, G}(X)\right)$ is derived asymptotically as $N \rightarrow \infty$. Let $U$ be a random variable uniformly distributed on $[-1 / 2,1 / 2]$.

Theorem 1. Let $X$ be a continuous random variable with density function $f(x), x \in \mathbb{R}$. Let $g(x)$ be a quantization density function and $Q_{N, G}(X)$ the non-uniform companding quantizer. If $\left(A_{1}\right)$ and $\left(A_{2}\right)$ hold, then

(i) $g(X) Z_{N, G}(X) \stackrel{d}{\rightarrow} U$ as $N \rightarrow \infty$;

(ii) $Z_{N, G}(X) \stackrel{d}{\rightarrow} U / g(X)$ as $N \rightarrow \infty$, where $X$ and $U$ are independent random variables.

Remark 1. In particular, this result is valid for random variables with values in a finite interval (cf. [13]).

Various quantization methods are used in quantization applications and theory. Now we consider a quantizer $\bar{Q}_{N, G}$ with the same quantization intervals as for $Q_{N, G}, I_{n, N}$, $n=1, \ldots, N$, but with quantization levels in the middle of the intervals, except for the intervals $I_{1, N}$ and $I_{N, N}$, where the levels do not change, i.e.,

$$
\bar{Q}_{N, G}(x)= \begin{cases}S\left(\frac{1}{2 N}\right) & \text { if } x \in I_{1, N}, \\ \frac{1}{2}\left(S\left(\frac{n}{N}\right)+S\left(\frac{n-1}{N}\right)\right) & \text { if } x \in I_{n, N}, n=2, \ldots, N-1, \\ S\left(\frac{2 N-1}{2 N}\right) & \text { if } x \in I_{N, N}\end{cases}
$$

(cf. [3]). Further, for a random variable $X$ we use the result from 9] demonstrating the asymptotic equivalence of the non-uniform quantizers $Q_{N, G}(X)$ and $\bar{Q}_{N, G}(X)$ with 
respect to the $r$ th-mean error distortion measure $D\left(Q_{N, G}\right)=\mathrm{E}\left|X-Q_{N, G}(X)\right|^{r}, r>0$, $N \geq 1$. For $X$ with density function $f(x)$ let $p(y)$ denote the probability density function of the random variable $Y:=G(X), p(y)=f(S(y)) / g(S(y)), y \in[0,1]$. Recall that $S$ is the inverse function of $G$. In particular, the assumption $\left(A_{2}\right)$ corresponds to boundedness of the density $p(y)$.

As in [9] we introduce the following sufficient conditions on $f(x)$ and $g(x)$ for the validity of (3):

$\left(B_{1}\right) g(x)$ is positive and continuous.

$\left(B_{2}\right)$ There exists $M>0$ such that $g(x)$ is increasing if $x<-M$ and $g(x)$ is decreasing if $x>M$.

$\left(B_{3}\right) \int_{\mathbb{R}} f(x) / g(x)^{r} d x<\infty$ for some $r>0$.

$\left(B_{4}\right)$ For some $\varepsilon>0$

$$
\int_{0}^{\varepsilon} s(x / 2)^{r} p(x) d x<\infty
$$

and

$$
\int_{1-\varepsilon}^{1} s((x+1) / 2)^{r} p(x) d x<\infty,
$$

where, by definition, $s(y)=S^{(1)}(y), y \in(0,1)$ (see also [2] for some different sufficient conditions on $f(x)$ and $g(x))$.

The assumption $\left(B_{1}\right)$ together with the tail conditions in $\left(B_{4}\right)$ is somewhat similar to $\left(A_{1}\right)$. Under the assumptions $\left(B_{1}\right)-\left(B_{4}\right)$, it is shown in 9] that the $r$ th-mean quantization errors for $N$-level non-uniform quantizers $Q_{N, G}$ and $\bar{Q}_{N, G}$ have the same behavior asymptotically as $N \rightarrow \infty$,

$$
\lim _{N \rightarrow \infty} N^{r} D\left(Q_{N, G}\right)=\lim _{N \rightarrow \infty} N^{r} D\left(\bar{Q}_{N, G}\right)=C_{r}>0
$$

(cf. (3)). This result justifies the application of the quantizer $Q_{N, G}$, at least with respect to the $r$ th-mean distortion measure. Moreover, in the following theorem we show that for a random variable $X$ the asymptotic normalized quantization errors

$$
Z_{N, G}(X)=N\left(X-Q_{N, G}(X)\right) \quad \text { and } \quad \bar{Z}_{N, G}(X)=N\left(X-\bar{Q}_{N, G}(X)\right)
$$

have identical stochastic structure as $N \rightarrow \infty$ under the assumptions $\left(A_{1}\right)-\left(A_{3}\right)$.

Theorem 2. Let $X$ be a continuous random variable with density function $f(x), x \in \mathbb{R}$. Let $g(x)$ be a quantization density function and $\bar{Q}_{N, G}(X)$ the non-uniform quantizer. If $\left(A_{1}\right)-\left(A_{3}\right)$ hold, then

(i) $g(X) \bar{Z}_{N, G}(X) \stackrel{d}{\rightarrow} U$ as $N \rightarrow \infty$.

(ii) $\bar{Z}_{N, G}(X) \stackrel{d}{\rightarrow} U / g(X)$ as $N \rightarrow \infty$, where $X$ and $U$ are independent random variables.

Remark 2. Theorems 1(ii) and 2(ii) correspond to the asymptotic result (3). In fact, $\mathrm{E}(U / g(X))^{r}=C_{r}$. These results can be applied when various asymptotic properties of quantization errors are investigated.

\section{Numerical EXAmPles AND Simulations}

The following numerical examples illustrate the rate of convergence in distribution for Theorems 1] and 2 from Section 2. In this section, the quantizers $Q_{N, G}$ and $\bar{Q}_{N, G}$ are said to be Quantizer 1 and 2, respectively. We approximate the distribution functions in the examples below by the corresponding empirical distribution functions. Denote by $F_{V}(x)$ the distribution function of a random variable $V$. Recall that $N$ denotes the number of quantization levels. Let $N_{s}$ denote the number of simulations. 
Example 1. Let $X_{1}$ be a standard Gaussian variable. Let $g_{1}(x)$ be a standard Gaussian quantization density function. The assumptions $\left(A_{1}\right)-\left(A_{3}\right)$ hold and we rewrite Theorems 1(i) and 2(i) in the following form:

$$
F_{g_{1}\left(X_{1}\right) Z_{N, G_{1}}\left(X_{1}\right)}(x) \rightarrow x+1 / 2 \quad \text { as } N \rightarrow \infty
$$

for Quantizer 1 and

$$
F_{g_{1}\left(X_{1}\right) \bar{Z}_{N, G_{1}}\left(X_{1}\right)}(x) \rightarrow x+1 / 2 \text { as } N \rightarrow \infty
$$

for Quantizer 2, where $G_{1}(x)$ is the distribution function corresponding to the density $g_{1}(x)$. Figures 1 and 2 illustrate the rate of convergence in (5) and (6), respectively.

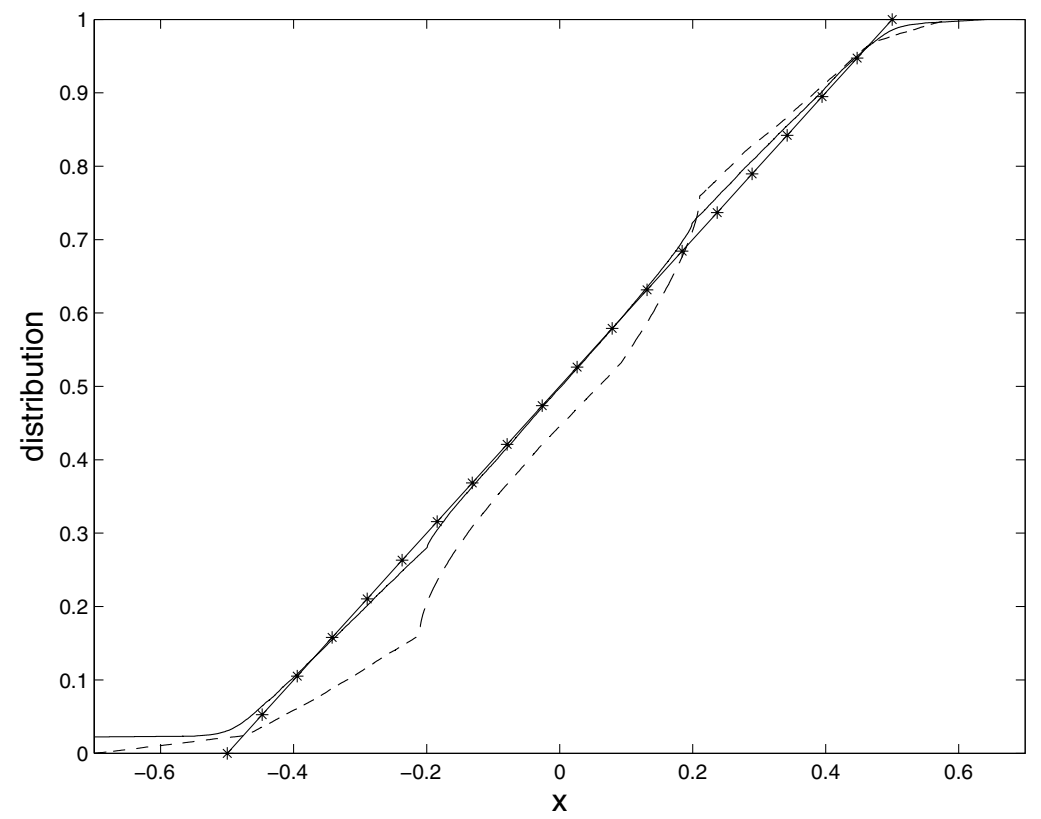

FIGURE 1. The empirical distribution functions $\widehat{F}_{g_{1}\left(X_{1}\right) Z_{N, G_{1}}\left(X_{1}\right)}(x)$ (Quantizer 1) for $N=3$ (dashed) and $N=20$ (solid), and the uniform distribution function $F_{U}(x)=x+1 / 2$ (stars); $N_{s}=50000$.

Example 2. Let $X_{2}$ be a Gaussian variable with zero mean and standard deviation 3 . Let $g_{2}(x)$ be a quantization density function of the Cauchy distribution with parameters 0 and 1. The assumptions $\left(A_{1}\right)-\left(A_{3}\right)$ hold and we rewrite Theorems 1 (ii) and 2(ii) in the following form:

$$
F_{Z_{N, G_{2}}\left(X_{2}\right)}(x) \rightarrow F_{U / g_{2}\left(X_{2}\right)}(x) \text { as } N \rightarrow \infty
$$

for Quantizer 1 and

$$
1 F_{\bar{Z}_{N, G_{2}}\left(X_{2}\right)}(x) \rightarrow F_{U / g_{2}\left(X_{2}\right)}(x) \quad \text { as } N \rightarrow \infty
$$

for Quantizer 2, where $G_{2}(x)$ corresponds to $g_{2}(x)$. Figures 3 and 4 illustrate the rate of convergence in (7) and (8), respectively. 


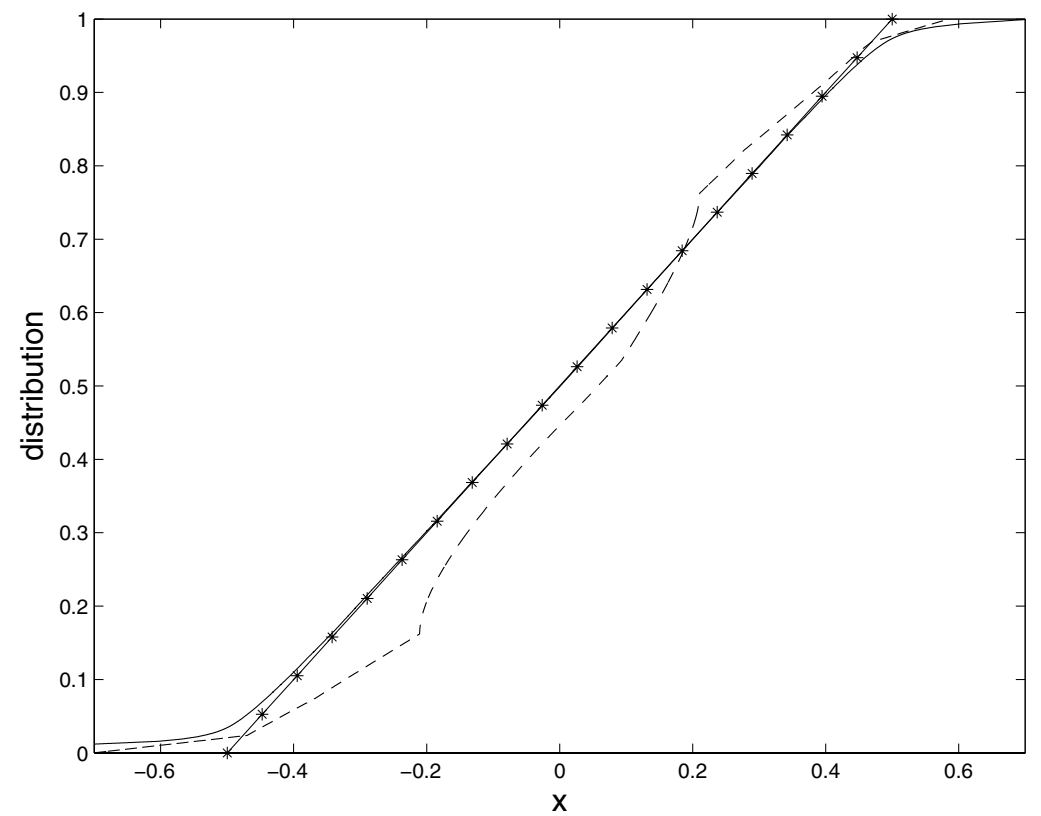

FIGURE 2. The empirical distribution functions $\widehat{F}_{g_{1}\left(X_{1}\right) \bar{Z}_{N, G_{1}}\left(X_{1}\right)}(x)$ (Quantizer 2) for $N=3$ (dashed) and $N=20$ (solid), and the uniform distribution function $F_{U}(x)=x+1 / 2$ (stars); $N_{s}=50000$.

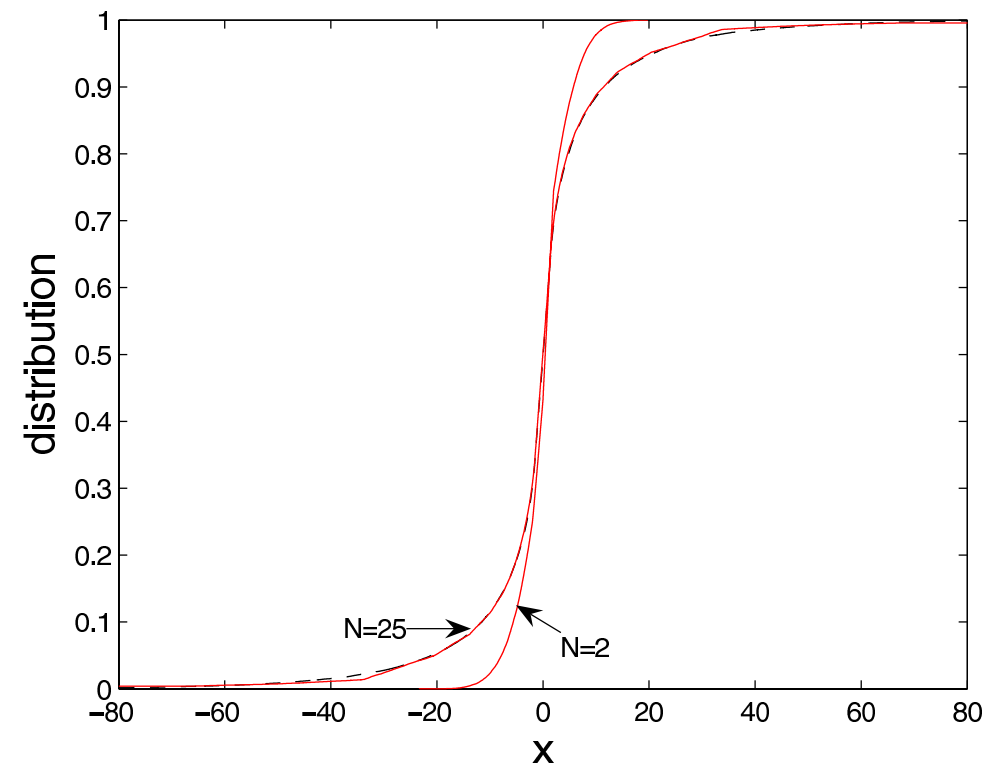

Figure 3. The empirical distribution functions $\widehat{F}_{Z_{N, G_{2}}\left(X_{2}\right)}(x)$ (Quantizer 1) for $N=2.25$ (solid), and $\widehat{F}_{U / g_{2}\left(X_{2}\right)}(x)$ (dashed); $N_{s}=50000$. 


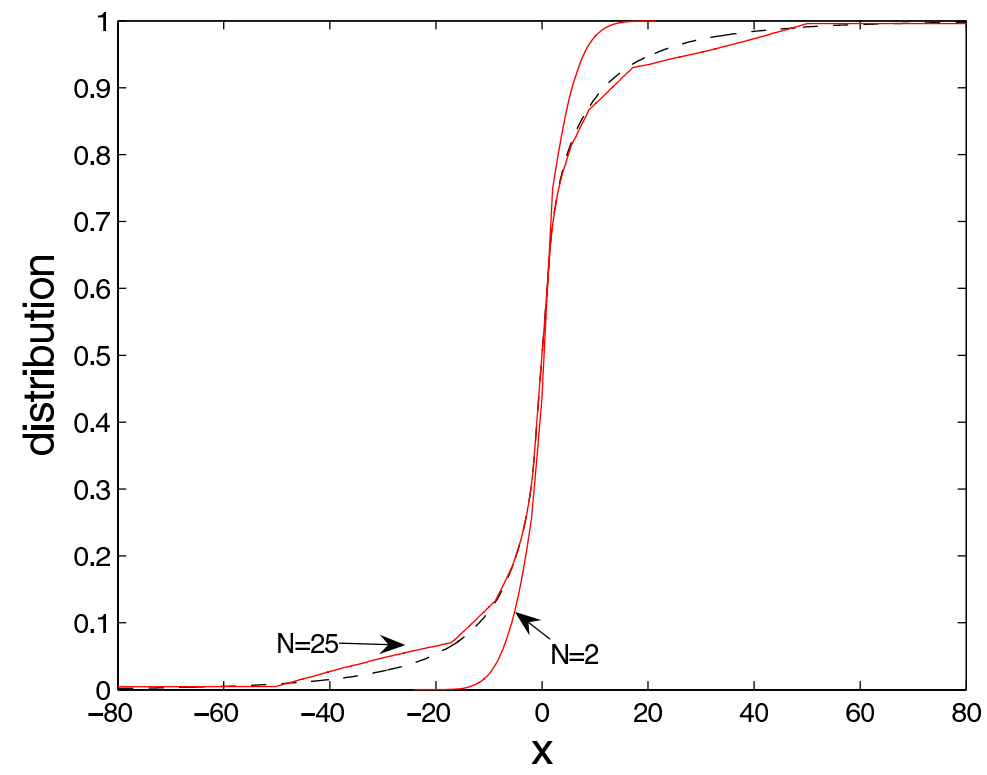

Figure 4. The empirical distribution functions $\widehat{F}_{\bar{Z}_{N, G_{2}}\left(X_{2}\right)}(x)$ (Quantizer 2) for $N=2.25$ (solid), and $\widehat{F}_{U / g_{2}\left(X_{2}\right)}(x)$ (dashed); $N_{s}=50000$.

\section{Proofs}

Henceforth, let $\phi_{V}(v)$ denote the characteristic function of a random variable $V$.

Proof of Theorem 1. We begin with (ii). For the random variable $Y=G(X)$ with values in $(0,1)$, we have the uniform $N$-level scalar quantizer

$$
Q_{N, U}(Y)=Q_{N, U}(G(X))=G\left(Q_{N, G}(X)\right)
$$

(cf. (11) and (2)), and the normalized quantization error $Z_{N, U}(Y)=N\left(Y-Q_{N, U}(Y)\right)$. Note that the function $G(x)$ is increasing and continuously differentiable, $G^{(1)}(x)=g(x)$, $x \in \mathbb{R}$. Thus the inverse function $S$ of $G$ is also continuously differentiable and

$$
S^{(1)}(y)=\frac{1}{g(S(y))}, \quad y \in(0,1) .
$$

By the mean value theorem, there exists $X_{N}$ between $X$ and $Q_{N, G}(X)$ such that

$$
Z_{N, U}(Y)=N\left(G(X)-G\left(Q_{N, G}(X)\right)\right)=g\left(X_{N}\right) Z_{N, G}(X) .
$$

We get

$$
Z_{N, G}(X)=\left(1+\frac{g\left(X_{N}\right)-g(X)}{g(X)}\right)^{-1} \frac{Z_{N, U}(Y)}{g(X)} .
$$

Further, uniform continuity of $g(x)$ (the assumption $\left(A_{1}\right)$ ) implies that

$$
\left(g\left(X_{N}\right)-g(X)\right) / g(X) \rightarrow 0 \quad \text { as } N \rightarrow \infty \quad \text { (a.s.). }
$$

Thus it follows by the Cramér-Slutsky Theorem that

$$
Z_{N, G}(X) \stackrel{d}{\rightarrow} U / g(X) \quad \text { as } N \rightarrow \infty
$$

since

$$
Z_{N, U}(Y) / g(X) \stackrel{d}{\rightarrow} U / g(X) \quad \text { as } N \rightarrow \infty
$$


$X=S(Y)$. In order to prove (10), we show that

$$
\phi_{Z_{N, U}(Y) / g(S(Y))}(v) \rightarrow \phi_{U / g(S(Y))}(v) \quad \text { as } N \rightarrow \infty, \quad v \in \mathbb{R} .
$$

By the definition (2), for the density function $p(y)$ of the random variable $Y$, we have

$$
\begin{aligned}
\phi_{Z_{N, U}(Y) / g(S(Y))}(v)=\int_{0}^{1} \exp \left\{i v N\left(y-Q_{N, U}(y)\right) / g(S(y))\right\} p(y) d y \\
=\sum_{n=1}^{N} \int_{(n-1) / N}^{n / N} \exp \left\{i v N\left(y-\left(\frac{n}{N}-\frac{1}{2 N}\right)\right) / g(S(y))\right\} p(y) d y \\
=\sum_{n=1}^{N} \int_{(n-1) / N}^{n / N-1 /(2 N)} \exp \left\{i v N\left(y-\left(\frac{n}{N}-\frac{1}{2 N}\right)\right) / g(S(y))\right\} p(y) d y \\
\quad+\sum_{n=1}^{N} \int_{n / N-1 /(2 N)}^{n / N} \exp \left\{i v N\left(y-\left(\frac{n}{N}-\frac{1}{2 N}\right)\right) / g(S(y))\right\} p(y) d y \\
=S_{1}(N)+S_{2}(N) .
\end{aligned}
$$

Consider the sum $S_{1}(N)$. We get

$$
\begin{aligned}
S_{1}(N)=\sum_{n=1}^{N} \int_{(n-1) / N}^{n / N-1 /(2 N)} \exp \left\{i v N\left(y-\left(\frac{n}{N}-\frac{1}{2 N}\right)\right) / g(S(y))\right\} p(y) d y \\
=\int_{-1 / 2}^{0}\left(\sum_{n=1}^{N} \exp \left\{i v z / g\left(S\left(\frac{1}{N}\left(n-\frac{1}{2}\right)+\frac{z}{N}\right)\right)\right\}\right. \\
\left.\times p\left((n-1 / 2) N^{-1}+z N^{-1}\right) N^{-1}\right) d z
\end{aligned}
$$

by changing the variable, $z=N\left(y-N^{-1}(n-1 / 2)\right)$. It follows from $\left(A_{2}\right)$ that there exists $C>0$ such that

$$
p(y)=f(S(y)) / g(S(y)) \leq C,
$$

and we get

$$
\left|\sum_{n=1}^{N} \exp \left\{i v z / g\left(S\left(\frac{1}{N}\left(n-\frac{1}{2}\right)+\frac{z}{N}\right)\right)\right\} p\left((n-1 / 2) N^{-1}+z N^{-1}\right) N^{-1}\right| \leq C .
$$

Hence, by the dominated convergence theorem and elementary properties of the Riemann integral,

$$
S_{1}(N) \rightarrow \int_{-1 / 2}^{0} \int_{0}^{1} e^{i v z / g(S(y))} p(y) d y d z \quad \text { as } N \rightarrow \infty .
$$

Similarly as for $S_{1}(N)$, we have

$$
S_{2}(N) \rightarrow \int_{0}^{1 / 2} \int_{0}^{1} e^{i v z / g(S(y))} p(y) d y d z \quad \text { as } N \rightarrow \infty .
$$

Now (12) together with (13) and (14) implies

$$
\phi_{Z_{N, U}(Y) / g(S(Y))}(v) \rightarrow \int_{-1 / 2}^{1 / 2} \int_{0}^{1} e^{i v z / g(S(y))} p(y) d y d z=\phi_{U / g(S(Y))}(v) \quad \text { as } N \rightarrow \infty
$$

and $S(Y)=X$ and $U$ are independent random variables. Hence (11) holds. This completes the proof of the assertion (ii).

The idea of the proof of (i) is the same as in the proof of (ii). Notice that

$$
Q_{N, G}(X)=S\left(Q_{N, U}(Y)\right), \quad X=S(Y) .
$$


Thus

$$
\begin{aligned}
g(X) Z_{N, G}(X) & =N g(X)\left(X-Q_{N, G}(X)\right) \\
& =N g(S(Y))\left(S(Y)-S\left(Q_{N, U}(Y)\right)\right) .
\end{aligned}
$$

Furthermore, it follows by the mean value theorem and (9) that there exists $Y_{N}$ between $Y$ and $Q_{N, U}(Y)$ such that $S(Y)-S\left(Q_{N, U}(Y)\right)=\left(Y-Q_{N, U}(Y)\right) / g\left(S\left(Y_{N}\right)\right)$. Therefore, combining $\left(A_{1}\right)$ with the Cramér-Slutsky Theorem applied to (15) and using that $g\left(S\left(Y_{N}\right)\right) \rightarrow g(S(Y))$ (a.s.) as $N \rightarrow \infty$ for $\left|Y_{N}-Y\right| \leq\left|Q_{N, U}(Y)-Y\right| \leq 1 / N$, we get

$$
g(X) Z_{N, G}(X)=\frac{g(S(Y))}{g\left(S\left(Y_{N}\right)\right)} Z_{N, U}(Y) \stackrel{d}{\rightarrow} U \quad \text { as } N \rightarrow \infty .
$$

In order to prove the assertion (i), it is enough to show that

$$
Z_{N, U}(Y) \stackrel{d}{\rightarrow} U \quad \text { as } N \rightarrow \infty
$$

or equivalently

$$
\phi_{Z_{N, U}(Y)}(v) \rightarrow \phi_{U}(v) \quad \text { as } N \rightarrow \infty, \quad v \in \mathbb{R} .
$$

But (16) can be proved in the same way as in the proof of (11). Hence (i) holds. This completes the proof of the theorem.

Proof of Theorem 2, First we prove (ii). By the definition we have

$$
\bar{Z}_{N, G}(X)=Z_{N, G}(X)+d_{N}(X)
$$

where $d_{N}(X)=d_{N, G}(X):=N\left(Q_{N, G}(X)-\bar{Q}_{N, G}(X)\right), N \geq 1$. Therefore, in view of the Cramér-Slutsky Theorem, to prove the assertion of the theorem it is enough to show that $d_{N}(X) \stackrel{d}{\rightarrow} 0$ or, equivalently,

$$
\phi_{d_{N}(X)}(v) \rightarrow 1 \quad \text { as } N \rightarrow \infty
$$

since, by Theorem 1, $Z_{N, G}(X) \stackrel{d}{\rightarrow} U / g(X)$ as $N \rightarrow \infty$. Observe that by the definitions

$$
\begin{aligned}
\phi_{d_{N}(X)}(v)= & \mathrm{E} e^{i v d_{N}(X)} \\
= & \sum_{n=2}^{N-1} \int_{S((n-1) / N)}^{S(n / N)} \exp \left\{i v N \left(S\left(\frac{n}{N}-\frac{1}{2 N}\right)\right.\right. \\
& \left.\left.-\frac{1}{2}\left(S\left(\frac{n}{N}\right)+S\left(\frac{n-1}{N}\right)\right)\right)\right\} f(x) d x .
\end{aligned}
$$

To prove (18) we estimate $\left|\phi_{d_{N}(X)}(v)-1\right|$ by splitting up the sum in (19). For a given $\delta>0$, select a sufficiently small $a_{0}=a_{0}(\delta)>0$ such that

$$
I_{2}\left(a_{0}\right):=\int_{0}^{a_{0}} p(y) d y+\int_{1-a_{0}}^{1} p(y) d y<\delta .
$$

We represent $\phi_{d_{N}(X)}(v)$ as follows:

$$
\begin{gathered}
\phi_{d_{N}(X)}(v)=S_{1, N}\left(a_{0}\right)+S_{2, N}\left(a_{0}\right), \\
1=I_{1}\left(a_{0}\right)+I_{2}\left(a_{0}\right),
\end{gathered}
$$


where

$$
\begin{aligned}
S_{1, N}\left(a_{0}\right):= & \sum_{\substack{2 \leq n \leq N-1: \\
n / N \in\left[a_{0}, 1-a_{0}\right]}} \exp \left\{i v \frac { N } { 2 } \left(\left(S\left(\frac{n}{N}-\frac{1}{2 N}\right)-S\left(\frac{n-1}{N}\right)\right)\right.\right. \\
& \left.\left.+\left(S\left(\frac{n}{N}-\frac{1}{2 N}\right)-S\left(\frac{n}{N}\right)\right)\right)\right\} \\
& \times \int_{S((n-1) / N)}^{S(n / N)} f(x) d x .
\end{aligned}
$$

Further, combining the mean value theorem with $\left(A_{3}\right)$ and (9), we get

$$
\begin{aligned}
S_{1, N}\left(a_{0}\right)=\sum_{\substack{2 \leq n \leq N-1: \\
n / N \in\left[a_{0}, 1-a_{0}\right]}} & \exp \left\{i \frac{v}{4}\left(\frac{1}{g\left(S\left(\nu_{n}^{\prime}\right)\right)}-\frac{1}{g\left(S\left(\nu_{n}^{\prime \prime}\right)\right)}\right)\right\} \\
& \times f\left(S\left(\omega_{n}^{\prime}\right)\right) \frac{1}{g\left(S\left(\omega_{n}^{\prime \prime}\right)\right)} \frac{1}{N}
\end{aligned}
$$

for some

$$
\nu_{n}^{\prime}, \nu_{n}^{\prime \prime}, \omega_{n}^{\prime}, \omega_{n}^{\prime \prime} \in[(n-1) / N, n / N], \quad n=2, \ldots, N-1 .
$$

We use the following elementary property of the Riemann integral: if two functions $h(x)$ and $\varphi(x)$ are Riemann integrable on $[a, b] \subset \mathbb{R}$, then

$$
\lim _{\max \left|\Delta x_{i}\right| \rightarrow 0} \sum_{i=0}^{n-1} h\left(\xi_{i}\right) \varphi\left(\theta_{i}\right) \Delta x_{i}=\int_{a}^{b} h(x) \varphi(x) d x,
$$

where

$$
x_{i} \leq \xi_{i}, \quad \theta_{i} \leq x_{i+1}, \quad \Delta x_{i}=x_{i+1}-x_{i}, \quad i=0,1, \ldots, n-1
$$

$\left(a=x_{0}<x_{1}<x_{2}<\cdots<x_{n}=b\right), n \geq 1$. This property can be generalized to any finite number of Riemann integrable functions. Thus (22) applied to functions

$$
\exp \left\{i \frac{v}{4}\left(\frac{1}{g(S(y))}-\frac{1}{g(S(y))}\right)\right\}
$$

$f(S(y))$, and $1 / g(S(y))$ in (21) implies that

$$
S_{1, N}\left(a_{0}\right) \rightarrow \int_{a_{0}}^{1-a_{0}} \frac{f(S(y))}{g(S(y))} d y=I_{1}\left(a_{0}\right) \quad \text { as } N \rightarrow \infty .
$$

Hence

$$
\left|S_{1, N}\left(a_{0}\right)-I_{1}\left(a_{0}\right)\right|<\delta
$$

for a sufficiently large $N$. We have also that

$$
\begin{aligned}
\left|S_{2, N}\left(a_{0}\right)\right| & \sum_{\substack{2 \leq n \leq N-1: \\
n / N \in[0,1] \backslash\left[a_{0}, 1-a_{0}\right]}} \int_{S((n-1) / N)}^{S(n / N)} f(x) d x \\
& \leq \int_{-\infty}^{S\left(a_{0}\right)} f(x) d x+\int_{S\left(1-a_{0}-1 / N\right)}^{\infty} f(x) d x \\
& =\int_{0}^{a_{0}} p(y) d y+\int_{1-a_{0}-1 / N}^{1} p(y) d y<2 \delta
\end{aligned}
$$


for a sufficiently large $N$, by changing the variable $y=G(x)$. Applying (20), (23), and (24), we obtain

$$
\left|\phi_{d_{N}(X)}(v)-1\right| \leq\left|S_{1, N}\left(a_{0}\right)-I_{1}\left(a_{0}\right)\right|+\left|S_{2, N}\left(a_{0}\right)\right|+\left|I_{2}\left(a_{0}\right)\right|<4 \delta
$$

for a sufficiently large $N$. Since $\delta>0$ is arbitrary, (18) holds. Using a similar technique as in the proof of (ii) and taking into account (17), Theorem[1(i), and (18), we immediately get the assertion (i). This completes the proof.

Acknowledgments. The author is grateful to Associate Professor Oleg Seleznjev for many fruitful discussions and suggestions during the work.

\section{BIBLIOGRAPHY}

1. W. R. Bennett, Spectrum of quantized signal, Bell. Syst. Tech. J. 27 (1948), 446-472. $\operatorname{MR} 0026287(10: 133 \mathrm{f})$

2. J. A. Bucklew and G. L. Wise, Multidimensional asymptotic quantization theory with rth power distortion measures, IEEE Trans. Inform. Theory 28 (1982), 239-247. MR651819 (83b:94026)

3. S. Cambanis and N. Gerr, A simple class of asymptotically optimal quantizers, IEEE Trans. Inform. Theory 29 (1983), 664-676. MR730904 (85a:94001)

4. S. Graf and H. Luschgy, Foundations of Quantization for Probability Distributions, SpringerVerlag, New York, 2000. MR1764176 (2001m:60043)

5. R. M. Gray and T. Linder, Mismatch in high rate entropy constrained vector quantization, IEEE Trans. Inform. Theory 49 (2003), 1204-1217. MR.1984821 (2004e:94026)

6. R. M. Gray and D. L. Neuhoff, Quantization, IEEE Trans. Inform. Theory 44 (1998), 23252383. MR 1658787 (99i:94029)

7. D. H. Lee and D. L. Neuhoff, Asymptotic distribution of the errors in scalar and vector quantizers, IEEE Trans. Inform. Theory 42 (1996), 446-460.

8. J. Li, N. Chaddha, and R. M. Gray, Asymptotic performance of vector quantizers with a perceptual distortion measure, IEEE Trans. Inform. Theory 45 (1999), 1082-1091. MR.1686244 (2000b:94008)

9. T. Linder, On asymptotically optimal companding quantization, Probl. Contr. Inform. Theory 20 (1991), 475-484.

10. H. Luschgy and G. Pagès, Functional quantization of Gaussian processes, Jour. Func. Annal. 196 (2002), 486-531. MR1943099 (2003i:60006)

11. O. Seleznjev, Spline approximation of random processes and design problems, Jour. Stat. Plan. Infer. 84 (2000), 249-262. MR1747507 (2001e:62082)

12. M. Shykula and O. Seleznjev, Uniform quantization of random processes, Univ. Umeå Research Report 1 (2004), 1-16.

13. M. Shykula and O. Seleznjev, Stochastic structure of asymptotic quantization errors, Stat. Prob. Letters 76 (2006), 453-464. MR.2266598

14. P. L. Zador, Asymptotic quantization error of continuous signals and the quantization dimensions, IEEE Trans. Inform. Theory 28 (1982), 139-148. MR651809 (83b:94014)

Department of Mathematics and Mathematical Statistics, Ume̊ University, SE-901 87 UMEÅ, SWEDEN

E-mail address: mykola.shykula@math.umu.se

Received 24/JUL/2005

Originally published in English 